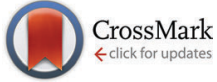

Cite this: Phys. Chem. Chem. Phys., 2016, 18, 29582

Received 29th July 2016, Accepted 27th September 2016

DOI: $10.1039 / c 6 c p 05285 a$

www.rsc.org/pccp

\title{
Modulation of the exfoliated graphene work function through cycloaddition of nitrile imines $\dagger$
}

\author{
Myriam Barrejón, ${ }^{a}$ María J. Gómez-Escalonilla, ${ }^{a}$ José Luis G. Fierro, ${ }^{b}$ \\ Pilar Prieto, ${ }^{c}$ José R. Carrillo, ${ }^{c}$ Antonio M. Rodríguez, ${ }^{c d}$ Gonzalo Abellán, ${ }^{e}$ \\ $M^{a}$ Cruz López-Escalante, ${ }^{f}$ Mercedes Gabás, ${ }^{g}$ Juan T. López-Navarrete ${ }^{h}$ and \\ Fernando Langa*a
}

\begin{abstract}
After the feasibility of the 1,3-dipolar cycloaddition reaction between nitrile imines and exfoliated graphene by density functional theory calculations was proved, very few-layer graphene was effectively functionalized using this procedure. Hydrazones with different electronic properties were used as precursors for the 1,3-dipoles, and microwave irradiation as an energy source enabled the reaction to be performed in a few minutes. The anchoring of organic addends on the graphene surface was confirmed by Raman spectroscopy, X-ray photoelectron spectroscopy (XPS) and thermogravimetric analysis. Ultraviolet photoelectron spectroscopy (UPS) was used to measure the work function and band gap of these new hybrids. Our results demonstrate that it is possible to modulate these important electronic valence band parameters by tailoring the electron richness of the organic addends and/or the degree of functionalization.
\end{abstract}

\section{Introduction}

Graphene is a two-dimensional material that is composed of a single layer of carbon atoms and it has recently attracted considerable scientific interest due to its significant physical properties and a large number of potential technological applications. ${ }^{1-3}$

${ }^{a}$ Universidad de Castilla-La Mancha, Instituto de Nanociencia,

Nanotecnología y Materiales Moleculares (INAMOL), 45071, Toledo, Spain. E-mail: Fernando.Langa@uclm.es

${ }^{b}$ Instituto de Catálisis y Petroleoquímica, CSIC, Cantoblanco, 28049, Madrid, Spain

${ }^{c}$ Departamento de Química Orgánica, Inorgánica y Bioquímica,

Facultad de Ciencias y Tecnologías Quimicas, Universidad de Castilla-La Mancha, Campus Universitario, 13071 Ciudad Real, Spain

${ }^{d}$ Dipartimento di Scienze Chimiche, Università degli Studi di Napoli Federico II, Via Cintia, 80126, Naples, Italy

${ }^{e}$ Department of Chemistry and Pharmacy and Institute of Advanced Materials and Processes (ZMP), Friedrich Alexander University Erlangen-Nürnberg, Henkestrasse, 42, 91054 Erlangen and Dr.-Mack Strasse 81, 90762 Fürth, Germany

${ }^{f}$ Unidad de Nanotecnología - The Nanotech Unit Dpto. Ingeniería Quimica, Lab. Materiales \& Superficies, Universidad de Málaga, 29071 Málaga, Spain

${ }^{g}$ Unidad de Nanotecnología - The Nanotech Unit Dpto. Física Aplicada I, Lab. Materiales \& Superficies, Universidad de Málaga, 29071 Málaga, Spain

${ }^{h}$ Department of Physical Chemistry, University of Málaga, Campus de Teatinos $s / n$, 29071, Málaga, Spain

$\dagger$ Dedicated to Prof. Tomas Torres on the occasion of his 65th birthday.

\# Electronic supplementary information (ESI) available: Additional data of TGA plots and Raman, XPS and FTIR data, AFM and HRTEM images, VBA spectra and also computational details. See DOI: 10.1039/c6cp05285a
Graphene is characterized as a zero band gap semimetal in which the conduction and the valence bands meet at the Dirac point. The zero bandgap nature of graphene results in a high current leakage, which limits its applications as a candidate in standard logic electronic circuits. In this regard, the introduction of a bandgap in graphene through band structure engineering $^{4-7}$ into a semiconductor by opening up the band gap, and thus enhancing its potential practical electronic applications is highly desirable. ${ }^{8,9}$ Covalent chemistry provides a powerful pathway to tailor the physical properties of pristine graphene to transform intrinsic zero band gap energy graphene. Based on the well-known covalent chemical reactivity of fullerenes and carbon nanotubes, chemists have already achieved good control of the elemental covalent chemistry of graphene and a broad arsenal of chemical reactions have already been carried out on this flat form of carbon, despite its low chemical reactivity when compared with curved carbon nanostructures such as fullerenes and carbon nanotubes. ${ }^{10-13}$ Cycloaddition chemistry is clearly an important tool in the chemistry of carbon nanostructures. The application of this chemistry leads to higher solubility, better control over composition and the development of more complicated carbon nanostructure architectures for novel properties and new applications. The most frequently employed type of cycloaddition in carbon nanostructure chemistry is arguably the 1,3-dipolar cycloaddition and, in particular, the cycloaddition of azomethine ylides. ${ }^{14,15}$ Nevertheless, nitrile imines, formed in situ in a one-pot procedure from hydrazones and $N$-bromosuccinimide (NBS) or 
$\mathrm{N}$-chlorosuccinimide (NCS) in the presence of triethylamine $\left(\mathrm{NEt}_{3}\right)$, react with fullerenes ${ }^{16}$ and carbon nanotubes ${ }^{17,18}$ as dipolarophiles under relatively mild reaction conditions to afford 2-pyrazoline derivatives in good yield. The scope of the reaction is very broad as hydrazones are readily available from aldehydes, which has allowed the preparation of a large number of derivatives for different practical purposes. It should be noted that with less reactive dipoles, the use of microwave irradiation (MWI) has proven to be very useful in cycloaddition reactions ${ }^{19,20}$ as well as in fullerene and carbon nanotube chemistry. ${ }^{21}$ The application of MWI allows the formation of cycloadducts in a few minutes. Significantly, in contrast to other fullerene derivatives, the resulting 2-pyrazolinefullerenes show better, or at least equivalent, electron affinity when compared to the parent $\mathrm{C}_{60}$ and they also exhibit remarkable thermal stability. ${ }^{22}$

Although numerous cycloaddition reactions have been explored experimentally with fullerenes ${ }^{23}$ or carbon nanotubes, ${ }^{24}$ and despite the fact that the covalent functionalization of graphene is a growing field of research, only the 1,3-dipolar cycloaddition of azomethine ylides ${ }^{14,15}$ has been applied to the functionalization of graphene. ${ }^{25-28}$

The band gap and the work function (WF) of a material provide information about the electronic states on the surface of the solid and most organic electronic devices require the use of semiconducting materials in which the work function should be adjustable, thus allowing efficient charge transport. In this sense, the possibility of adjusting the work function through chemical functionalization makes graphene a very promising material in the semiconductor field. ${ }^{29}$ Band-gap opening has been demonstrated after in situ doping, ${ }^{30}$ chemical doping, ${ }^{31}$ electrochemical doping $^{32}$ or $\mathrm{BN}$ doping of chemical vapor deposited graphene. ${ }^{33}$ The WF of graphene can be tuned by using different methods, such as the electric field effect, ${ }^{34}$ chemical doping with heteroatoms, ${ }^{35}$ or chemical functionalization. ${ }^{36-38}$ However, the effect of surface functionalization on the electronic structure of graphene is still not well understood, and systematic studies on the variation of the graphene WF reported as a function of the type of functionalization are rare. ${ }^{32,39}$ Reduced graphene oxide (rGO) has also attracted attention as an alternative to achieve easily fabricated organic graphene-based optoelectronic devices. Theoretical studies using density functional theory (DFT) calculations on the tunability of rGO predict an increase in the WF of up to $2.5 \mathrm{eV}$ through the precise control of oxygen-containing functional groups. ${ }^{31}$ Ultraviolet laser irradiation has been used to increase the WF of chlorinated GO layers by up to $5.23 \mathrm{eV}^{40}$

Based on our experience with fullerenes and carbon nanotube chemistry, in the present work we assessed theoretically and experimentally the feasibility of the cycloaddition of four different $N$ - $p$-nitrophenylnitrile imines onto the surface of graphene. The hybrid materials were fully characterized and a systematic study was performed using ultraviolet photoelectron spectroscopy (UPS) on the modulation of the graphene work function by modifying the electron richness of the pyrazoline substituent and the degree of functionalization. This contribution aims to offer a new and reliable route to achieve controlled graphene band engineering.

\section{Experimental section}

General procedure for the synthesis of graphene pyrazolines (3a-d) under classical heating $(\mathrm{CH})$

Pyridine $(0.5 \mathrm{~mL})$ was added to a solution of the appropriate hydrazone $(0.10 \mathrm{mmol})$ in dry chloroform $\left(\mathrm{CHCl}_{3}\right)(15 \mathrm{~mL})$ under argon and the mixture was cooled to $0{ }^{\circ} \mathrm{C}$. NCS $(0.42 \mathrm{mmol}, 56 \mathrm{mg})$ was added and the mixture was stirred for 30 minutes. Thereafter, chloroform was evaporated under vacuum and then a solution of exfoliated graphene (EG) in $N$-methylpyrrolidone (NMP) $(80 \mathrm{~mL})$ and triethylamine $(2 \mathrm{~mL})$ were added and the resulting solution was heated at $170{ }^{\circ} \mathrm{C}$ under argon for 6 days. The crude product was separated by centrifugation, decanted, and then washed several times with methanol and dichloromethane.

\section{General procedure for the synthesis of graphene-pyrazolines} (3a-d) under microwave conditions (MWI)

Pyridine $(0.5 \mathrm{~mL})$ was added to a solution of the appropriate hydrazone $(0.10 \mathrm{mmol})$ in dry $\mathrm{CHCl}_{3}(15 \mathrm{~mL})$ under argon and the mixture was cooled to $0{ }^{\circ} \mathrm{C}$. NCS $(0.42 \mathrm{mmol}, 56 \mathrm{mg})$ was added and the mixture was stirred for 30 minutes. Thereafter, chloroform was evaporated under vacuum and then a solution of exfoliated graphene (EG) in NMP $(80 \mathrm{~mL})$ and triethylamine ( $2 \mathrm{~mL}$ ) were added in a microwave glass vessel and the mixture was subjected to microwave irradiation $\left(40 \mathrm{~W}, 170{ }^{\circ} \mathrm{C}, 1\right.$ hour $)$. After cooling to room temperature, the crude product was separated by centrifugation, decanted, and then washed several times with methanol and dichloromethane.

Preparation of 3a. According to both general procedures, pyridine $(0.5 \mathrm{~mL})$, hydrazone (1a) $(28 \mathrm{mg}, 0.10 \mathrm{mmol})$, NCS (56 mg, $0.42 \mathrm{mmol})$, exfoliated graphene in NMP $(80 \mathrm{~mL})$ and triethylamine $(2 \mathrm{~mL})$ gave the desired product.

Preparation of $\mathbf{3 b}$. According to both general procedures, pyridine $(0.5 \mathrm{~mL})$, hydrazone (1b) $(25 \mathrm{mg}, 0.10 \mathrm{mmol})$, NCS (56 mg, $0.42 \mathrm{mmol})$, exfoliated graphene in NMP $(80 \mathrm{~mL})$ and triethylamine $(2 \mathrm{~mL})$ gave the desired product.

Preparation of 3c. According to both general procedures, pyridine $(0.5 \mathrm{~mL})$, hydrazone (1c) $(37 \mathrm{mg}, 0.10 \mathrm{mmol})$, NCS (56 mg, $0.42 \mathrm{mmol})$, exfoliated graphene in NMP $(80 \mathrm{~mL})$ and triethylamine $(2 \mathrm{~mL})$ gave the desired product.

Preparation of 3d. According to both general procedures, pyridine $(0.5 \mathrm{~mL})$, hydrazone $(1 \mathrm{~d})(22 \mathrm{mg}, 0.10 \mathrm{mmol})$, NCS (56 mg, $0.42 \mathrm{mmol})$, exfoliated graphene in NMP $(80 \mathrm{~mL})$ and triethylamine $(2 \mathrm{~mL})$ gave the desired product.

Preparation of 4. $70 \mathrm{~mL}$ of the graphene dispersion in NMP (7.13 mg) together with sarcosine $(48 \mathrm{mg}, 0.54 \mathrm{mmol})$ and 2-thiophenecarboxaldehyde ( $60 \mathrm{mg}, 0.54 \mathrm{mmol}$ ) were heated by irradiation for 1 hour at $40 \mathrm{~W}$ and $170{ }^{\circ} \mathrm{C}$. The mixture was then brought to room temperature. Separation by centrifugation and washing with methanol and dichloromethane afforded the desired material.

\section{Results and discussion}

\section{Theoretical study}

To better understand the reactivity, regioselectivity and stereoselectivity of the 1,3-dipolar cycloaddition of nitrile imines to 
graphene we carried out a computational study with DFT calculations. In the last decade, the scientific community has followed different pathways for the computational description of the reactivity of graphene. ${ }^{41}$ One of these approaches involves the description of graphene as a two dimensional infinite superstructure by $a b$ initio methods within periodic boundary conditions. $^{42,43}$ This approach is employed in order to determine the electronic properties of graphene and its derivatives and it allows the study of the organized monolayer adsorption of molecules on the surface. The description of graphene as a 'finite' structure is an approach that is widely used in the recent literature on graphene and it correctly describes the interaction with isolated molecules. This is also the best approach to describe the chemical reactivity due to its low computational cost. $^{44-46}$ For this reason, in this work we employed finite-size polybenzenoid ( 25 fused benzene rings) hydrocarbons as graphene models and did not consider defects.

Previous studies by Houk ${ }^{47,48}$ indicate that polyacenes have an open-shell singlet ground state. ${ }^{49}$ This polyradical behavior was also found in graphene nanoflakes with systems that were around 100 carbon atoms in size. ${ }^{50}$ It was found that graphene models are more stable as open-shell singlet states than as closed-shell singlet and open-shell triplet states. ${ }^{51}$ For this purpose, all calculations reported here were performed using Gaussian 09 (see details in the ESI $\ddagger$ ) using the (U) M06-2X level of theory ${ }^{52,53}$ and $6-31 \mathrm{G}(\mathrm{d})$ basis set ${ }^{54-58}$ as this method is reported to provide excellent energy values for cycloaddition reactions. ${ }^{59}$ This approach therefore takes into account the dispersion interactions because the $\mathrm{sp}^{2}$ framework of graphene can interact through $\pi$-stacking and $\mathrm{CH}-\pi$ interactions with the dipolarophile. All stationary points were characterized by harmonic analysis. All relative energies reported in this paper include zero-point vibrational energy (ZPVE) corrections. Both models, i.e., zigzag (model 1) and armchair (model 2) edges, terminated with hydrogen atoms were considered ${ }^{47,48}$ (Scheme 1).

Firstly, the reactant structures were optimized and the topologies and energy values of the frontier molecular orbitals (see Fig. S1, ESI $\ddagger$ ) and the band gap were calculated. All of the outcomes are collected in Table S1 (ESI $\$$ ). The band gap energy values are referenced to graphene sheet model 2 as it was determined computationally that this is $28.67 \mathrm{kcal} \mathrm{mol}^{-1}$ more stable than model 1 . The calculations indicate that the orbital energy gaps are low enough to allow the reaction; as depicted in Table S1 (ESI ), for nitrile imines $2 \mathbf{b}, \mathbf{c}$ the energy difference between the HOMO of the graphene and the LUMO of the nitrile imines is smaller than that between the LUMO of the graphene and the HOMO of the nitrile imine - a finding that suggests an inverse electron demand approach; in the case of nitrile imines $\mathbf{2 a}$ and $\mathbf{2 d}$ both approaches are possible.

Next, we computed the reaction path considering tertbutylnitrile imine 2d and both graphene models. To our knowledge there are few examples in the literature in which the transition states have been computed. ${ }^{44-48}$ The DFT computational time scale is about $n^{4}$ ( $n=$ number of atoms) and it is more complex in the case of transition states. The reactivity of the graphene model described in the literature is justified only

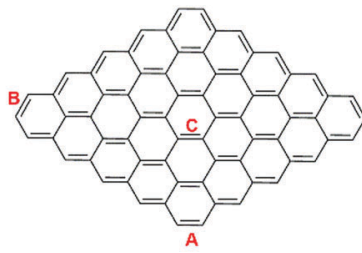

Model 1

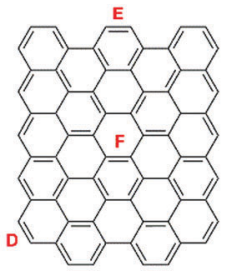

Model 2

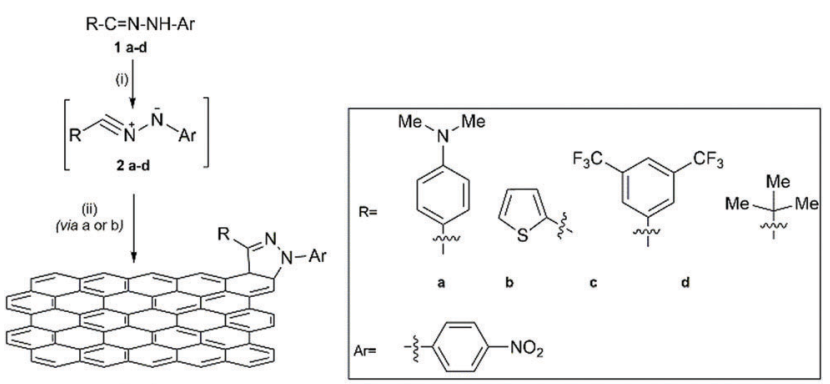

3a.d

Scheme 1 (top): Graphene models and bonds considered. A to E denote the possible positions of attack in the reaction. (bottom): 1,3-Dipolar cycloaddition between nitrile imines $\mathbf{2 a - d}$, formed in situ from hydrazones 1a-d and graphene (EG). Reagents and conditions: (i) NCS, pyridine, $\mathrm{CHCl}_{3}$, $0{ }^{\circ} \mathrm{C}$ to RT, $30 \mathrm{~min}$; (ii) EG, NMP, NEt, $170{ }^{\circ} \mathrm{C}$, (a) classical heating, 6 days or (b) microwave irradiation, $40 \mathrm{~W}, 1 \mathrm{~h}$.

for thermodynamic reasons. As a consequence, we considered the kinetics as well as the regioselectivity and the stereoselectivity of this process. Thus, we computed the complete reaction path for six possible attacks, which are denoted as A, B, C, D, E and F (Scheme 1, top). For each case, two possible regioisomers were considered and, within each, both possible stereoisomers were assessed. These are denoted as follows: subscript $\mathbf{1}$ is employed for graphene model 1 , subscript 2 is employed for graphene model 2; r1, r2, c1 and c2 denote nitro groups right, left, ahead and behind, respectively. Hence, the four possible approaches (except for functionalization in positions $\mathbf{C}$ and $\mathbf{F}$, in the center of the sheet) are denoted as r1c1, r1c2, r2c1, and r2c2, where r1c1, r2c1 and r1c2, r2c2 are enantiomers (see Fig. S2 (ESI $\$$ ) for clarity). The outcomes are collected in Table 1.

The results clearly indicate that the attack on the central positions ( $\mathbf{C}$ and $\mathbf{F}$ ) is unfavorable under both kinetic (27.5 and $38.8 \mathrm{kcal} \mathrm{mol}^{-1}$ ) and thermodynamic (17.2 and $6.7 \mathrm{kcal} \mathrm{mol}^{-1}$ ) considerations (Table 1, entries 9 and 18). Conversely, reactions on the corner and the edge bonds will be favored kinetically and thermodynamically. It is noteworthy that c2 attack is always kinetically favored due to a $\pi-\pi$ interaction between the nitrobenzene ring and the graphene sheet in the transition states (TS) (Fig. 1).

In contrast, the $\mathbf{c} 1$ attack is thermodynamically favoured. In all these cases the products show obvious curvature originating from the tetrahedral atoms at which the cycloaddition occurs. This distortion decreases the steric interactions (Fig. 1). Thus, it can be concluded that the 1,3-dipolar cycloaddition of nitrile imines to graphene may proceed from the kinetic and thermodynamic points of view. The most reactive positions, both kinetically and thermodynamically, are $\mathbf{A}$ and $\mathbf{D}$; considering that 
Table 1 Activation energies $\left(\Delta E_{\mathrm{a}}, \mathrm{kcal}^{\mathrm{mol}}{ }^{-1}\right)$ and reaction energies $\left(\Delta E_{\mathrm{rxn}}, \mathrm{kcal} \mathrm{mol}^{-1}\right)$ computed at the (U)M06-2X/6-31G(d) level

\begin{tabular}{|c|c|c|c|}
\hline Entry & Attack & $\Delta E_{\mathrm{a}}\left(\mathrm{Kcal} \mathrm{mol}^{-1}\right)$ & $\Delta E_{\mathrm{rxn}}\left(\mathrm{kcal} \mathrm{mol}^{-1}\right)$ \\
\hline 1 & $A_{1}-\mathbf{r} 1 \mathrm{c} 1$ & 10.2 & -47.5 \\
\hline 2 & $A_{1}-r 1 c 2$ & 3.5 & -42.1 \\
\hline 3 & $\mathrm{~A}_{1}-\mathrm{r} 2 \mathrm{c} 1$ & 10.2 & -47.5 \\
\hline 4 & $A_{1}-\mathbf{r} 2 c 2$ & 3.9 & -38.2 \\
\hline 5 & $\mathrm{~B}_{1}-\mathrm{r} 1 \mathrm{c} 1$ & 14.8 & -30.6 \\
\hline 6 & $\mathrm{~B}_{1}-\mathrm{r} 1 \mathrm{c} 2$ & 9.7 & -23.5 \\
\hline 7 & $\mathrm{~B}_{1}-\mathrm{r} 2 \mathrm{c} 1$ & 14.9 & -30.4 \\
\hline 8 & $B_{1}-\mathrm{r} 2 \mathrm{c} 2$ & 9.5 & -23.6 \\
\hline 9 & C & 27.5 & 17.2 \\
\hline 10 & $D_{2}-\mathrm{r} 1 \mathrm{c} 1$ & 10.6 & -44.1 \\
\hline 11 & $D_{2}-\mathrm{r} 1 \mathrm{c} 2$ & 4.6 & -33.5 \\
\hline 12 & $D_{2}-\mathbf{r} 2 \mathrm{c} 1$ & 11.9 & -44.2 \\
\hline 13 & $D_{2}-\mathbf{r} 2 \mathrm{c} 2$ & 5.7 & -29.6 \\
\hline 14 & $E_{2}-\mathbf{r} 1 \mathrm{c} 1$ & 18.8 & -11.2 \\
\hline 15 & $E_{2}-\mathbf{r} 1 \mathrm{c} 2$ & 9.1 & -12.6 \\
\hline 16 & $E_{2}-\mathbf{r} 2 \mathrm{c} 1$ & 18.4 & -20.2 \\
\hline 17 & $E_{2}-\mathrm{r} 2 \mathrm{c} 2$ & 9.1 & -9.1 \\
\hline 18 & $\mathbf{F}$ & 38.8 & 6.7 \\
\hline
\end{tabular}
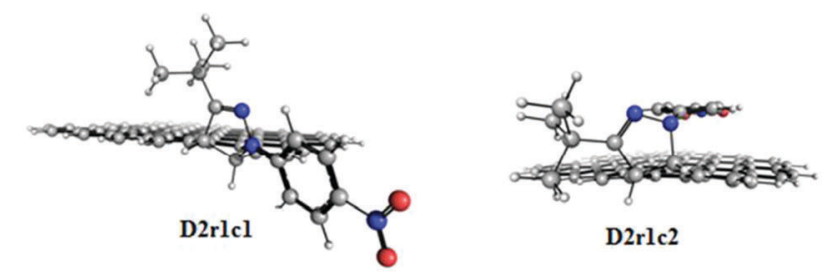

D2rlc2
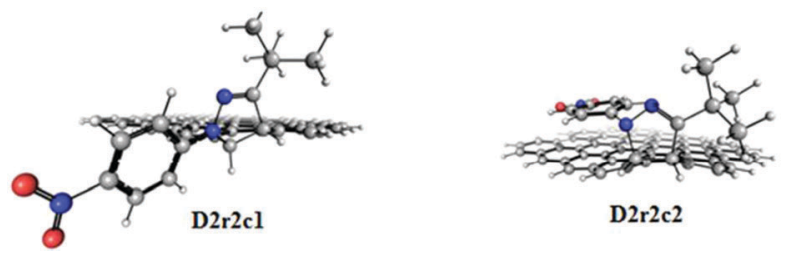

Fig. 1 The four possible stereoisomers resulting from attack on position $\mathbf{D}$ (the most favorable) computed at the (U) M06-2X/6-31G(d) theory level.

graphene model 2 (armchair) is $28 \mathrm{kcal} \mathrm{mol}^{-1}$ more stable than model 1, position $\mathbf{D}$ (in the corner) would be the most favoured.

\section{Experimental demonstration}

Having confirmed by computational studies the feasibility of the reaction, we proceeded with the experimental verification by reacting exfoliated graphene with several nitrile imines generated in situ from hydrazones functionalized with different substituents: electron donors (4- $N, N$-dimethylamino, thiophene), ${ }^{60}$ a moderate donor (tert-butyl) ${ }^{61}$ and an electron acceptor (3,5-bistrifluoromethylphenyl). ${ }^{62}$

Few-layer graphene was obtained by sonication of graphite flakes in $N$-methylpyrrolidone (NMP) (see the ESI $\ddagger$ ). ${ }^{63}$ Analysis of the Raman spectrum (laser excitation at $532 \mathrm{~nm}$ ) of exfoliated graphene (EG) showed that the 2D band can be deconvoluted into three bands (Fig. 2 and Fig. S3, ESI $\ddagger$ ) (Lorentzian-shaped peaks) that are characteristic of few-layer graphene (less than 5 layers). ${ }^{64}$ The $I_{2 \mathrm{D}} / I_{\mathrm{G}}$ ratio of 0.5 is indicative of a three/four layer graphene structure. ${ }^{65,66}$ Further insights into the nature of EG were obtained by atomic force microscopy (AFM),

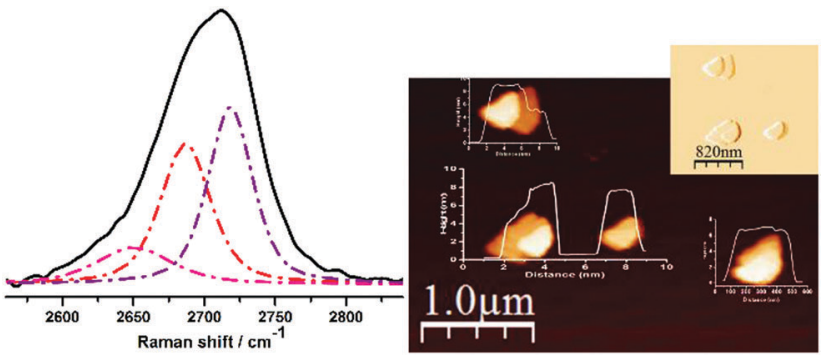

Fig. 2 (left) Raman spectrum of the 2D band region deconvoluted into three Lorentzian peaks (532 nm: laser excitation energy); (right) AFM image of an EG solution deposited onto $\mathrm{SiO}_{2}$ (the inset shows the presence of overlapped few layers graphene with an average height of ca. $4 \mathrm{~nm}$ ).

which revealed flakes with an average height of $c a .4 \mathrm{~nm}$, thus confirming the exfoliation process in 3-4 layers (Fig. 2 right and Fig. S4, ESI ).

The study of the pristine exfoliated graphene by transmission electron microscopy (TEM) in $N, N$-dimethylformamide (DMF) showed the presence of some aggregates of graphene platelets that were several hundreds of nanometers in the lateral dimensions. Fig. S5A-C (ESI $\$$ ) show the presence of a residual amorphous contribution, probably arising from hydrocarbon contamination and/or DMF solvent, as recently reported by Kaiser et al. ${ }^{67}$ A detailed inspection of some of the thinnest flakes revealed that the aggregates consist of highly crystalline few-layer graphene that on average are less than $5 \mathrm{~nm}$ in thickness - a finding that is consistent with the AFM studies. Indeed, Fig. S5D (ESI ) exhibits the characteristic fringes with a thickness of $c a .2 .8 \mathrm{~nm}$ and this is consistent with approximately 8 graphene layers.

We proceeded to carry out covalent functionalization with nitrile imines as dipoles prepared in situ from hydrazones. Pyrazolinefunctionalized graphene hybrids 3a-d were prepared in a one-pot procedure from hydrazones 1a-d and NCS in the presence of $\mathrm{NEt}_{3}$ and these were reacted with EG for 6 days at $170{ }^{\circ} \mathrm{C}$ (Scheme 1, bottom). ${ }^{16,68}$ Microwave heating methods have emerged as an interesting alternative in fullerene, ${ }^{69}$ carbon nanotube ${ }^{70}$ and graphene chemistry; ${ }^{71}$ therefore, this source of energy was used in these reactions and the reaction time was reduced to 1 hour.

Besides, in order to compare the influence of the nature of functionalization on the work function of graphene (vide infra), pyrrolidino graphene $\mathbf{4}$ was prepared by reaction of exfoliated graphene with 2-formylthiophene and $N$-methylglycine in NMP at $170{ }^{\circ} \mathrm{C}$ for 1 hour under microwave irradiation (see details in the ESI $\ddagger$ and Scheme $\mathrm{S} 1$ ).

The structural and thermal properties of the new graphenebased nanohybrids 3a-d and $\mathbf{4}$ were investigated by Raman and X-ray photoelectron spectroscopy (XPS), thermogravimetric analysis (TGA) and infrared spectroscopy (FTIR). AFM and HR-TEM studies were also performed for morphological characterization.

Confirmation of the covalent functionalization was obtained by Raman spectroscopy with laser excitation at $532 \mathrm{~nm}$. As shown in Fig. 3 (top) and Fig. S6 (ESI $\ddagger$ ), the ratio $I_{\mathrm{D}} / I_{\mathrm{G}}$, which shows minimal disorder in EG $\left(I_{\mathrm{D}} / I_{\mathrm{G}}=0.05\right)$, increased markedly in 3a-d 

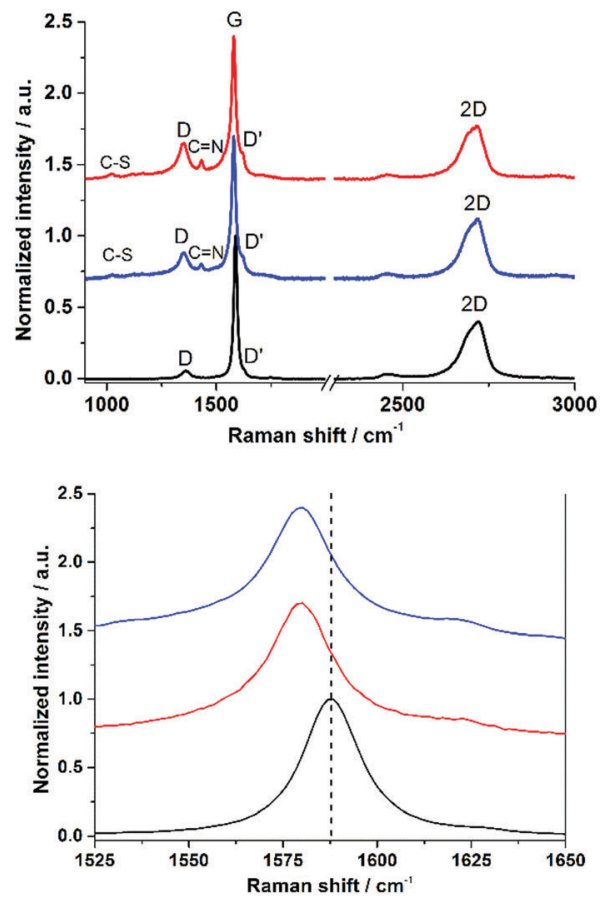

Fig. 3 (top): Raman spectra of $\mathbf{3 b}$ compared with that of EG (in black). The spectrum in blue represents the hybrid prepared by classical heating and in red under microwave irradiation. $\lambda_{\text {exc }}=532 \mathrm{~nm}$. (bottom): G-band region $\left(\lambda_{\text {exc }}=532 \mathrm{~nm}\right)$ of $E G$ (black line) in comparison with nanohybrid $\mathbf{3 b}$ (blue line by classical heating and red line under microwave irradiation).

(the $I_{\mathrm{D}} / I_{\mathrm{G}}$ ratio ranged from 0.15 to 0.32 , see Table 22 , ESI $\ddagger$ ) due to the increase in the number of $\mathrm{sp}^{3}$ carbons that were formed on the graphene during functionalization. More importantly, in 3a-d a peak $(\mathrm{C}=\mathrm{N})$ was observed at approximately $1430 \mathrm{~cm}^{-1}$ and this provides evidence of the formation of the pyrazole ring on the exfoliated graphene material. ${ }^{72}$

Furthermore, the $\mathrm{NO}_{2}$ band, which overlaps with the $\mathrm{G}$ band, was observed at around $1590 \mathrm{~cm}^{-1}$. In the case of the thiophene derivative $3 \mathbf{b}$ a peak at $1020 \mathrm{~cm}^{-1}$ corresponding to $\mathrm{C}-\mathrm{S}$ aromatic vibration was also present. Finally, it should be noted that, for all pyrazoline-graphene derivatives $\mathbf{3 a - d}$, the $\mathrm{G}$ band was shifted to lower frequencies $\left(\nu=10 \mathrm{~cm}^{-1}\right)$ and this provides evidence of n-doping of the material ${ }^{73-75}$ (Fig. 3 bottom).

The chemical environment of the functional groups and their concentration on the graphene surface were evaluated by photoelectron spectroscopy (XPS). ${ }^{76,77}$ As can be observed from the results in Table 2, the experimental and the theoretically

Table 2 Comparison of the surface atomic ratios of functionalized graphene (samples $\mathbf{3 a - d}$ and $\mathbf{4}$ ) prepared under classical heating $(\mathrm{CH})$ and microwave irradiation (MWI)

\begin{tabular}{|c|c|c|c|c|c|c|c|c|c|c|}
\hline \multirow[b]{2}{*}{ Sample } & \multicolumn{2}{|c|}{$\mathrm{C}(\mathrm{at} \%)$} & \multicolumn{2}{|c|}{$\mathrm{O}(\mathrm{at} \%)$} & \multicolumn{2}{|c|}{$\mathrm{N}(\mathrm{at} \%)$} & \multicolumn{2}{|c|}{ S (at $\%)$} & \multicolumn{2}{|c|}{ F (at\%) } \\
\hline & $\mathrm{CH}$ & MWI & $\mathrm{CH}$ & MWI & $\mathrm{CH}$ & MWI & $\mathrm{CH}$ & MWI & $\mathrm{CH}$ & MWI \\
\hline $3 \mathbf{a}$ & 95.5 & 95.6 & 4.1 & 3.9 & 0.4 & 0.5 & - & - & - & - \\
\hline $3 \mathbf{b}$ & 95.4 & 96.1 & 4.2 & 3.5 & 0.3 & 0.3 & 0.1 & 0.1 & - & - \\
\hline $3 c$ & 95.4 & 95.5 & 4.0 & 3.6 & 0.2 & 0.3 & - & - & 0.4 & 0.6 \\
\hline $3 d$ & 96.2 & 95.8 & 3.1 & 3.8 & 0.7 & 0.4 & - & - & - & - \\
\hline 4 & - & 96.1 & - & 3.6 & - & 0.5 & - & 0.5 & - & - \\
\hline
\end{tabular}

expected values of N/S and N/F atomic ratios for samples $3 \mathbf{b}$ and 3c (prepared under classical heating or microwave irradiation) are almost the same, thus providing further evidence for the covalent anchoring of the pyrazoline moiety on the graphene sheets. Details of the XPS study can be found in the ESI $¥$ (Fig. S7-S10).

Further evidence for the 1,3-dipolar cycloaddition on the surface of graphene was obtained by comparing the TGA curves of EG with those of the functionalized graphene materials 3a-d (see Fig. S11 (ESI\$) for TGA profiles of nanohybrids 3a-d and 4). The weight loss observed for EG $(2.6 \%)$ is attributed to the defects caused by sonication, ${ }^{78}$ which are also evident in the Raman spectra (D/G band ratio) (see Fig. S3, ESI $\$$ ). All functionalized graphene materials obtained by classical heating showed an additional loss in the temperature range $250-550{ }^{\circ} \mathrm{C}$ due to thermal decomposition of the covalently grafted organic groups on the graphene. Similar weight losses were found for the samples obtained under microwave irradiation, although the reaction time in this case was reduced from 6 days (under classical heating) to 1 hour (Table S3, ESI $\ddagger$ ).

The anchoring of the pyrazoline ring onto the EG surface was also verified by FTIR spectroscopy (Fig. S12, ESI $\ddagger$ ). Due to the formation of the pyrazoline ring, the $\mathrm{NH}$ bond stretching at 3250-3300 $\mathrm{cm}^{-1}$ corresponding to the hydrazone group was absent from the spectra of the functionalized graphene samples 3a-d. In addition, new features attributed to the aromatic $\mathrm{C}=\mathrm{C}$ stretching were observed between 1500 and $1400 \mathrm{~cm}^{-1}$ after the cycloaddition reaction. It is also important to note the peak observed at $690 \mathrm{~cm}^{-1}$ in the spectrum corresponding to the graphene derivative $\mathbf{3 b}$ (Fig. S12, ESI $\$$ ), which is attributed to the $\mathrm{C}-\mathrm{S}$ stretching of the thiophene ring. ${ }^{79}$

The functionalized graphene hybrids were further characterized by carrying out morphological studies by means of TEM (3c and 3d) and AFM (3b) techniques. AFM images and height profiles of graphene reveal the presence of few-layer graphene with an average height of $c a .4 \mathrm{~nm}$, which is in good agreement with the Raman results. Fig. S4 (ESI ) shows a structure with several height levels (terraces) and this suggests the collapse of two exfoliated flakes, i.e., the dispersed graphene tends to aggregate readily during the deposition process. ${ }^{80}$ The small heights of the pyrazoline rings mean that a similar situation was evident from the AFM image of $3 \mathbf{b}$ (Fig. 4).

In order to gain some insight into the microstructure of the materials, HRTEM experiments were performed on selected
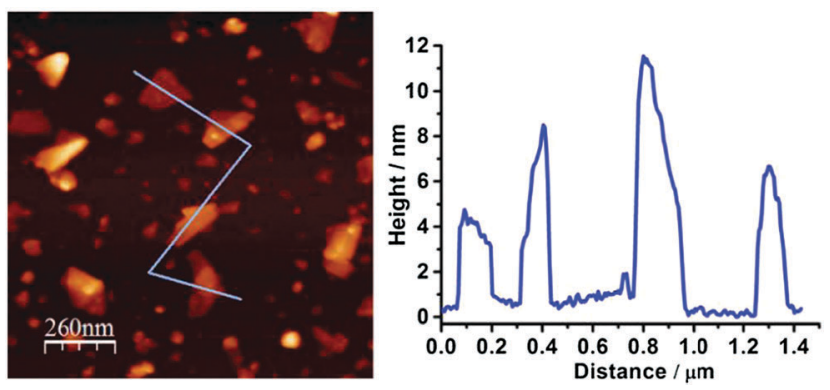

Fig. 4 AFM image of sample $3 \mathbf{b}$. The height profile is taken along the solid line. 


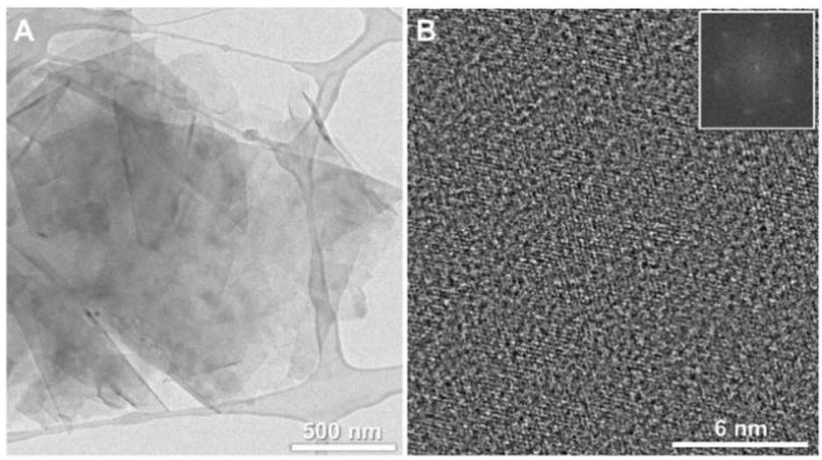

Fig. 5 (A) Low magnification image of functionalized sample $\mathbf{3 d}$ deposited onto a holey carbon TEM grid. (B) HRTEM (300 kV) image of a functionalized graphene sheet showing that the crystal lattice retains its hexagonal pattern (see inset). This situation was also corroborated by the presence of a hexagonal symmetry in the fast Fourier transform (FFT). The observation of several defects and dislocations, as well as amorphous areas, suggests the presence of pyrazoline moieties.

samples (3c and $\mathbf{3 d}$ ). Suspensions in ethanol of samples $\mathbf{3 c}$ and 3d were drop cast onto a lacey carbon grid and desiccated at $75{ }^{\circ} \mathrm{C}$ under vacuum. The HRTEM results for functionalized sample 3d (tert-butyl derivative) are shown in Fig. 5 and Fig. S13 (ESI†). It can be seen from Fig. 5A that the aggregate structure consists of few-layer graphene after the functionalization process. Interestingly, a higher magnification of the thinnest flakes revealed the typical crystalline hexagonal lattice along with the contribution of some amorphous moieties, which can be related to the presence of the pyrazolines and/or some physisorbed organic solvent residues. The analysis of the functionalized sample $\mathbf{3 c}$ revealed a markedly different morphology consisting of aggregates of smaller flakes, thus highlighting the influence of the fluoroalkyl groups (Fig. S14, ESI‡).

\section{Work function measurements}

Finally, ultraviolet photoelectron spectroscopy (UPS) was employed to explore the band structure of the graphene-based nanohybrids. The spectra show similarities and differences between the EG reference and the functionalized derivatives (see the ESI\$). The work function $(\mathrm{WF})$ data for the graphene-functionalized derivatives are represented in Fig. 6.

Functionalization decreases the graphene $\mathrm{WF}$ in all cases but to different extents depending on the nature of the radical and on the degree of functionalization (C atoms/organic group). The addition of electron-donating organic groups further decreases the $\mathrm{WF}$ values, i.e., 4.45 and $4.46 \mathrm{eV}$ for graphene-based nanohybrids $\mathbf{3 a}$ and $\mathbf{3 b}$. In contrast, electron-acceptor organic groups (samples 3c) led to an increase in the WF values ( 4.82 and $4.64 \mathrm{eV}$, depending on the degree of functionalization) when compared to those of derivatives $3 \mathrm{~d}$ and $\mathbf{4}$ (4.59 and $4.54 \mathrm{eV}$, respectively), thus making the WF closer to that of the EG (see green lines in Fig. 6). Higher functionalization (551 $\mathrm{C}$ atoms/organic group) led to a lower WF value $(4.64 \mathrm{eV})$, while lower functionalization ( $803 \mathrm{C}$ atoms/organic group) gave a value of $4.82 \mathrm{eV}$. This evidence strongly supports our hypothesis on the functionalization degree as a key parameter in WF tunability, but the results for $\mathbf{3 c}$ samples
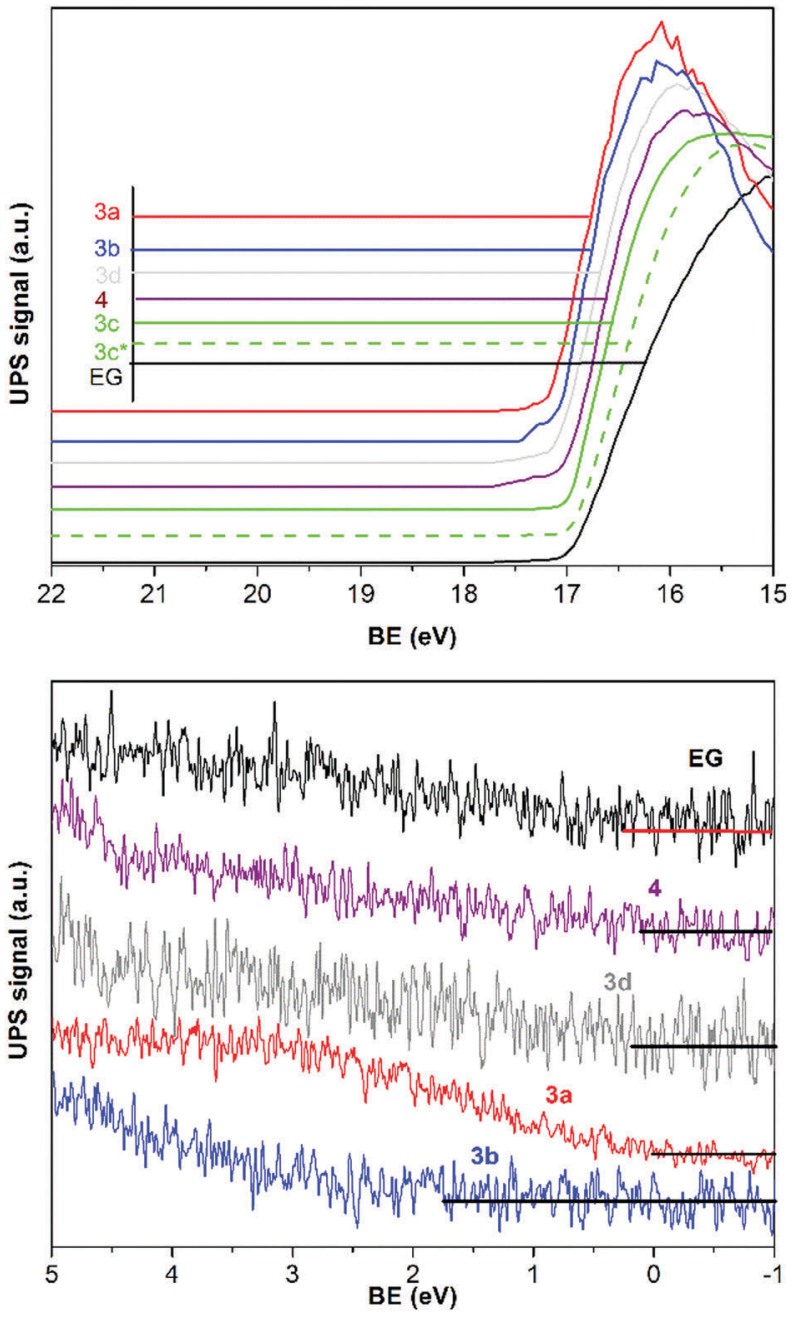

Fig. 6 (top) WF determination for graphene-based derivatives. EG, black line; $3 c$, green lines: solid and dashed lines corresponding to higher and lower functionalization degrees, respectively; 4, purple line; $\mathbf{3 d}$, gray line; 3b, blue line; $3 a$, red line. (bottom) VBE for the graphene-based derivatives. $\mathrm{EG}$, black line; $\mathbf{4}$, purple line, $\mathbf{3} \mathbf{d}$, gray line, $\mathbf{3} \mathbf{a}$, red line; $\mathbf{3 b}$, blue line. Horizontal lines are a guide to the eye. For the sake of clarity, spectra have been displaced.

also demonstrate the role of the organic group in the WF for these graphene derivatives.

The assumption outlined above was supported by $\mathbf{3 d}$ and $\mathbf{4}$ (pyrazoline vs. pyrrolidine derivative), in which the functionalization degree was very similar (see Fig. 7), where the values of the WF were also comparable $(4.59 \mathrm{eV} v s .4 .54 \mathrm{eV})$. This fact supports the hypothesis that the functionalization degree is one of the parameters that must be managed in order to control WF engineering in these graphene derivatives.

On the other hand, different results were also observed depending on the kind of functionalization (pyrazoline vs. pyrrolidine). Comparison of the effect on the work function after the functionalization of graphene with an analogous pyrrolidine derivative 4 (see Scheme S1, ESI ) shows a more marked decrease in graphene WF in the case of the pyrazoline derivative, which could be attributed to the presence of the lone pair of nitrogen 


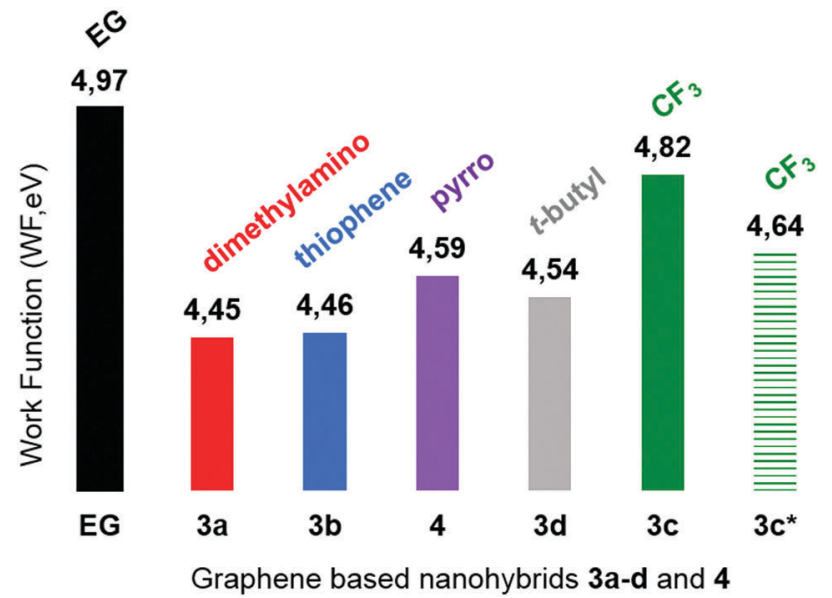

Fig. 7 Values of the WF (eV) for EG and graphene-based nanohybrids 3a-d and 4. * Higher degree of functionalization.

electrons located very close to the surface of graphene. This lone pair is absent in the case of the pyrrolidine derivative.

On the other side of the spectra, the valence band edge (VBE) was evaluated for all of the graphene-based nanohybrids (Fig. 6, bottom). Acceptor organic groups are not expected to affect the VBE since they would shift the LUMO orbitals, i.e. the band conduction edge, which cannot be evaluated by photoelectron spectroscopy. Functionalization itself does not have a noticeable effect on the VBE of graphene-based derivatives. The VBE of graphene derivatives $\mathbf{3 d}$ and $\mathbf{4}$ do not differ significantly from that of the EG sample $(\sim 0 \mathrm{eV})$. The incorporation of a donor organic group leads to a very clear difference related to the type of organic group and not to the degree of functionalization since this was very similar for derivatives $3 \mathbf{a}$ and $\mathbf{3 b}$ (Fig. 6 bottom). While the presence of the $N, N$-dimethylamino moiety (3a) does not have any effect on the VBE position, the incorporation of thiophene (3b) clearly opens a gap in this graphene-based nanohybrid. The VBE position is estimated to be $1.7 \mathrm{eV}$. This is a very surprising result given the similar donor character of the derivatives with $N, N$-dimethylamino and thiophene.

Moreover, it is worth mentioning here that pyrrolidine derivative $\mathbf{4}$ also contains a thiophene group and a band gap was not opened in this derivative.

These results must be compared with the Raman spectra measured for these samples, in particular with the G-band shift detected for the pyrazoline derivatives functionalized with donor radicals and for the pyrrolidine-thiophene derivative, which indicates the n-type doping of these nanohybrids. ${ }^{73-75}$ However, it seems that in spite of the similar donor character of $\mathrm{N}, \mathrm{N}$-dimethylamino and thiophene, only the link between thiophene and pyrazoline is able to achieve an effective band gap opening. According to the Raman spectra, the G-band shift for the pyrrolidine derivative $\mathbf{4}$ is half that of pyrazoline derivatives (see Fig. 3 and Fig. S6, ESI $\$$ ). This fact is also indicative of the influence of the type of functionalization on the work function.

The values obtained for the WF $(4.46 \mathrm{eV})$ and band gap $(1.7 \mathrm{eV})$ in the case of the $\mathbf{3 b}$ nanohybrid are rather remarkable when compared with some other published results. The findings of this study highlight ${ }^{34}$ this kind of graphene derivative as one of the most promising candidates to build graphene-based organic devices.

\section{Conclusions}

In summary, we have studied computationally and experimentally the 1,3-dipolar cycloaddition of nitrile imines to graphene. Although the reaction is slow under classical heating, the use of microwave irradiation allows the reaction to be carried out in a few minutes. Finally, we show that it is possible to modulate the band gap and the work function by modifying the electronic properties of the organic addend or the degree of functionalization.

\section{Acknowledgements}

Financial support from Mineco (Spain) (CTQ2013-48252-P and CTQ2015-71936-REDT), Junta de Comunidades de Castilla-La Mancha (PEII-2014-014-P) and the University of Castilla-La Mancha (UCLM) (GI20163531) is gratefully acknowledged. One of us (MB) thanks the FPI for a grant from Mineco. G. A. thanks the EU for a Marie Curie Fellowship (FP/2013-IEF-627386) and Dr F. Hauke and Prof. A. Hirsch for their kind support with the HRTEM equipment. Technical support from High Performance Computing Service of the University of Castilla-La Mancha is gratefully acknowledged.

\section{Notes and references}

1 K. S. Novoselov, A. K. Geim, S. V. Morozov, D. Jiang, Y. Zhang, S. V. Dubonos, I. V. Grigorieva and A. A. Firsov, Science, 2004, 306, 666-669.

2 A. K. Geim, Nat. Chem., 2007, 6, 183-191.

3 A. H. Castro Neto, F. Guinea, N. M. R. Peres, K. S. Novoselov and A. K. Geim, Rev. Mod. Phys., 2009, 81, 109-162.

4 L. A. Chernozatonskii, D. G. Kvashnin, O. P. Kvashnina and N. A. Konstantinova, J. Phys. Chem. C, 2014, 118, 1318-1321.

5 S. Bae, S. J. Kim, D. Shin, J.-H. Ahn and B. H. Hong, Phys. Scr., 2012, T146, 014024.

6 T.-H. Han, Y. Lee, M.-R. Choi, S.-H. Woo, S.-H. Bae, B. H. Hong, J.-H. Ahn and T.-W. Lee, Nat. Phononics, 2012, 6, 105-110.

7 K. C. Kwon, K. S. Choi and S. Y. Kim, Adv. Funct. Mater., 2012, 22, 4724-4731.

8 A. K. Geim, Science, 2009, 324, 1530-1534.

9 K. S. Mali, J. Greenwood, J. Adisoejoso, R. Phillipson and S. De Feyter, Nanoscale, 2015, 7, 1566-1585.

10 A. Criado, M. Melchionna, S. Marchesan and M. Prato, Angew. Chem., Int. Ed., 2015, 54, 10734-10750.

11 J. M. Englert, C. Dotzer, G. Yang, M. Schmid, C. Papp, J. M. Gottfried, H.-P. Steinrück, E. Spiecker, F. Hauke and A. Hirsch, Nat. Chem., 2011, 3, 279-286.

12 S. Eigler and A. Hirsch, Angew. Chem., Int. Ed., 2014, 53, 7720-7738. 
13 G. L. C. Paulus, Q. H. Wang and M. S. Strano, Acc. Chem. Res., 2013, 46, 160-170.

14 M. Maggini, G. Scorrano and M. Prato, J. Am. Chem. Soc., 1993, 115, 9798-9799.

15 M. Prato and M. Maggini, Acc. Chem. Res., 1998, 31, 519-601.

16 J. L Delgado, N. Martín, P. de la Cruz and F. Langa, Chem. Soc. Rev., 2011, 40, 5232-5241.

17 M. Álvaro, P. Atienzar, P. de la Cruz, J. L. Delgado, H. García and F. Langa, J. Phys. Chem. B, 2004, 108, 12691-12697.

18 M. Álvaro, P. Atienzar, P. de la Cruz, J. L. Delgado, V. Troiani, H. García, F. Langa, A. Palkar and L. Echegoyen, J. Am. Chem. Soc., 2006, 128, 6626-6635.

19 A. de la Hoz, A. Loupy, K. Bougrin and R. Benhid, Microwaves in Organic Synthesis; "Microwave-Assisted Cycloaddition Reactions", Wiley-VCH, 2013, ch. 17, vol. 1.

20 P. Appukkuttan, V. P. Mehta and E. V. van der Eychen, Chem. Soc. Rev., 2010, 39, 1467-1477.

21 F. Langa and P. de la Cruz, Microwaves in Organic Synthesis; "Application of Microwave Irradiation in Carbon Nanostructures", Wiley-VCH, 2013, ch. 22.

22 J. L. Delgado, F. Oswald, F. Cardinali, F. Langa and N. Martín, J. Org. Chem., 2008, 73, 3184-3188.

23 F. Langa and J. F. Nierengarten, "Fullerenes: Principles and Applications", RSC Publishing, 2012.

24 N. Karousis, N. Tagmatarchis and D. Tasis, Chem. Rev., 2010, 110, 5366-5397.

25 V. Georgakilas, "Functionalization of Graphene”, John Wiley \& Sons, 2014.

26 L. Rodríguez-Pérez, M. A. Herranz and N. Martín, Chem. Commun., 2013, 49, 3721-3735.

27 D. García, L. Rodríguez-Pérez, M. A. Herranz, D. Peña, E. Guitián, S. Bailey, Q. Al-Galiby, M. Noori, C. J. Lambert, D. Pérez and N. Martín, Chem. Commun., 2016, 52, 6677-6680.

28 V. Georgakilas, M. Otyepka, A. B. Bourlinos, V. Chandra, N. Kim, K. C. Kemp, P. Hobza, R. Zboril and K. S. Kim, Chem. Rev., 2012, 112, 6156-6214.

29 R. Garg, N. K. Dutta and N. R. Choudhury, Nanomaterials, 2014, 4, 267-300 and references therein.

30 J. Park, W. C. Mitchel, G. J. Brown, S. Elhamri, L. Grazulis, H. E. Smith, S. D. Pacley, J. J. Boeclk, K. G. Eyink, S. Mou, D. H. Tomich and J. E. Hoelscher, Appl. Phys. Lett., 2011, 98, 203102.

31 H. Liu, Y. Liu and D. Zhua, J. Mater. Chem., 2011, 21, 3335-3345.

32 C. S. Park, Y. Zhao, J.-H. Lee, D. Whang, Y. Shon, Y.-H. Shong and C. J. Lee, Appl. Phys. Lett., 2013, 102, 032106.

33 C.-K. Chang, S. Kataria, C.-C. Kuo, A. Ganguly, B.-Y. Wang, J.-Y. Hwang, K.-J. Huang, W.-H. Yang, W.-H. Yang, S.-B. Wang, C.-H. Chuang, M. Chen, C.-I. Huang, W.-F. Pong, K.-J. Song, S.-J. Chang, J.-H. Guo, Y. Tai, M. Tsujimoto, S. Isoda, C.-W. Chen, L.-C. Chen and K. Chen, ACS Nano, 2013, 7, 1333-1341.

34 Y. J. Yu, Y. Zhao, S. Ryu, L. E. Brus, K. S. Kim and P. Kim, Nano Lett., 2009, 9, 3430-3434.

35 Y. Shin, K. K. Kim, M. Hofmann, L. J. Li and J. Kong, ACS Nano, 2010, 4, 2689-2694.
36 Y.-J. Kim, Y. Kim, K. Novoselov and B. H. Hong, 2D Mater., 2015, 2, 042001.

37 P. V. Kumar, M. Bernardi and J. C. Grossman, ACS Nano, 2013, 7, 1638-1645.

38 B. Kang, S. Lim, W. H. Lee, S. B. Jo and K. Cho, Adv. Mater., 2013, 25, 5856-5862.

39 S.-T. Han, Y. Zhou, P. Sonar, H. Wei, L. Zhou, Y. Yan, C.-S. Lee and A. L. Roy, ACS Appl. Mater. Interfaces, 2015, 7, 1699-1708.

40 E. Stratakis, K. Savva, D. Konios, C. Petridisa and E. Kymakis, Nanoscale, 2014, 6, 6925-6931.

41 M. Pykal, P. Jurečka, F. Karlický and M. Otyepka, Phys. Chem. Chem. Phys., 2016, 18, 6351-6372.

42 P. A. Denis and F. Iribarne, Int. J. Quantum Chem., 2010, 110, 1764-1771.

43 P. A Denis and F. Iribarne, J. Mater. Chem., 2012, 22, 5470-5477.

44 V. León, A. M. Rodríguez, P. Prieto, M. Prato and E. Vázquez, ACS Nano, 2014, 1, 563-571.

45 Y. Cao and K. N. Houk, J. Mater. Chem., 2011, 21, 1503-1508. 46 Y. Yuan, P. Chen, X. Ren and H. Wang, ChemPhysChem, 2012, 13, 741-750.

47 Y. Cao, S. Osuna, Y. Liang, R. C. Haddon and K. N. Houk, J. Am. Chem. Soc., 2013, 135, 17643-17649.

48 P. A. Denis, Chem. - Eur. J., 2013, 15719-15725.

49 M. Bendikov, H. M. Doung, K. Starkey, K. N. Houk, E. A. Carter and F. Wuld, J. Am. Chem. Soc., 2004, 126, 7416-7417.

50 F. Plasser, H. Pasalic, M. H. Gerzabek, F. Libish, R. Reiter, J. Burgdorfer, T. Müller, R. Shepard and H. Lischka, Angew. Chem., Int. Ed., 2013, 52, 2581-2584.

51 S. Bian, A. M. Scott, Y. Cao, Y. Liang, S. Osuna, K. N. Houk and A. B. Braunschweig, J. Am. Chem. Soc., 2013, 135, 9240-9243.

52 Y. Zhao and D. G. Truhlar, Theor. Chem. Acc., 2008, 120, 215-241.

53 Y. Zhao and D. G. Truhlar, Acc. Chem. Res., 2008, 41, 157-167.

54 R. Ditchfield, W. J. Hehre and J. A. Pople, J. Chem. Phys., 1971, 54, 724-728.

55 W. J. M. Hehre, R. Ditchfield and J. A Pople, J. Chem. Phys., 1972, 56, 2257-2261.

56 P. C. Hariharan and J. A. Pople, Theor. Chim. Acta, 1973, 28, 213-222.

57 P. C. Hariharan and J. A. Pople, Mol. Phys., 1974, 27, 209-215. 58 M. S. Gordon, Chem. Phys. Lett., 1980, 76, 163-168.

59 Y. Lan, L.-F. Zou, Y. Cao and K. N. Houk, Phys. Chem. Chem. Phys., 2008, 10, 6615-6620.

60 E. Espíldora, J. L. Delgado, P. de la Cruz, A. de la Hoz, V. López-Arza and F. Langa, Tetrahedron, 2002, 58, 5821-5826.

61 J. L. Delgado, S. Filippone, A. Martín-Domenech, M. Altable, E. Maroto, F. Langa, N. Martín and R. Martínez-Alvarez, J. Am. Soc. Mass Spectrom., 2011, 22, 557-567.

62 J. L. Delgado, P. de la Cruz, V. López-Arza, F. Langa, Z. Gan, Y. Araki and O. Ito, Bull. Chem. Soc. Jpn., 2005, 78, 1500-1507.

63 Y. Hernández, V. Nicolosi, M. Lotya, F. M. Blighe, Z. Sun, S. De, I. T. McGovern, B. Holland, M. Byrne, Y. K. Gunko, J. J. Boland, P. Niraj, G. Duesberg, S. Krishnamurthy, R. Goodhue, J. Hutchison, V. Scardaci, A. C. Ferrari and J. N. Coleman, Nat. Nanotechnol., 2008, 3, 563-568. 
64 A. C. Ferrari, J. C. Meyer, V. Scardaci, C. Casiraghi, M. Lazzeri, F. Mauri, S. Piscane, D. Jiang, K. S. Novoselov, S. Roth and A. K. Geim, Phys. Rev. Lett., 2006, 97, 187401.

65 S. Sarkar, E. Bekyarova and R. C. Haddon, Mater. Today, 2012, 15, 276-285.

66 Y. Y. Wang, Z. H. Ni, T. Yu, H. M. Wang, Y. H. Wu, W. Chen, A. T. S. Wee and Z. X. Shen, J. Phys. Chem. C, 2008, 112, 10637-10640.

67 G. Algara-Siller, O. Lehtinen, A. Turchanin and U. Kaiser, Appl. Phys. Lett., 2014, 104, 153115.

68 F. Langa and P. de la Cruz, Comb. Chem. High Throughput Screening, 2007, 10, 766-782 and reference herein.

69 F. Langa, P. de la Cruz, E. Espíldora, J. J. García, M. C. Pérez and A. de la Hoz, Carbon, 2000, 38, 1642-1646.

70 A. Criado, M. Vizuete, M. J. Gómez-Escalonilla, S. GarcíaRodriguez, J. L. G. Fierro, A. Cobas, D. Peña, E. Guitián and F. Langa, Carbon, 2013, 63, 140-148.

71 M. Barrejón, A. Primo, M. J. Gómez-Escalonilla, J. L. García-Fierro, H. García and F. Langa, Chem. Commun., 2015, 51, 16916-16919.

72 M. Vizuete, M. J. Gómez-Escalonilla, J. L. G. Fierro, P. Atienzar, H. García and F. Langa, ChemPhysChem, 2014, 15, 100-108.
73 V. Straub, A. Gallego, G. de la Torre, T. W. Chamberlain, A. N. Khlobystov, T. Torres and D. M. Guldi, Faraday Discuss., 2014, 173, 233-256.

74 R. Voggu, C. S. Rout, A. D. Franklin, T. S. Fisher and C. N. R. Rao, J. Phys. Chem. C, 2008, 34, 13053-13056.

75 L. M. Arellano, L. Martín-Gomis, H. B. Gobeze, M. Barrejón, D. Molina, M. J. Gómez-Escalonilla, J. L. G. Fierro, M. Zhang, M. Yudasaka, S. Iijima, F. D’Souza, F. Langa and Á. SastreSantos, J. Mater. Chem. C, 2015, 3, 10215-10224.

76 M. Vizuete, M. J. Gómez-Escalonilla, J. L. G. Fierro, K. Ohkubo, S. Fukuzumi, M. Irie, M. Yudasaka, S. Iijima, J.-F. Nierengarten and F. Langa, Chem. Sci., 2014, 5, 2072-2080.

77 M. Barrejón, M. Vizuete, M. J. Gómez-Escalonilla, J. L. G. Fierro, I. Berlanga, F. Zamora, G. Abellán, P. Atienzar, J.-F. Nierengarten, H. García and F. Langa, Chem. Commun., 2014, 50, 9053-9055.

78 M. Quintana, J. I. Tapia and M. Prato, Beilstein J. Nanotechnol., 2014, 5, 2328-2338.

79 M. S. Teker, U. Tamer and N. Ö. Pekmez, Synth. Met., 2012, 162, 924-930.

80 Z. Jin, T. P. McNicholas, C.-J. Shih, Q. H. Wang, G. L. C. Paulus, A. J. Hilmer, S. Shimizu and M. S. Strano, Chem. Mater., 2011, 23, 3362-3370. 